Prepared for the U.S. Department of Energy under Contract DE-AC05-76RL01830

\title{
Effect of Background Emissivity on Gas Detection in Thermal Hyperspectral Imagery
}

\author{
SJ Walsh LK Chilton \\ MF Tardiff CN Metoyer
}

July 2008 


\title{
DISCLAIMER
}

This report was prepared as an account of work sponsored by an agency of the United States Government. Neither the United States Government nor any agency thereof, nor Battelle Memorial Institute, nor any of their employees, makes any warranty, express or implied, or assumes any legal liability or responsibility for the accuracy, completeness, or usefulness of any information, apparatus, product, or process disclosed, or represents that its use would not infringe privately owned rights. Reference herein to any specific commercial product, process, or service by trade name, trademark, manufacturer, or otherwise does not necessarily constitute or imply its endorsement, recommendation, or favoring by the United States Government or any agency thereof, or Battelle Memorial Institute. The views and opinions of authors expressed herein do not necessarily state or reflect those of the United States Government or any agency thereof.

\author{
PACIFIC NORTHWEST NATIONAL LABORATORY \\ operated by \\ BATTELLE \\ for the \\ UNITED STATES DEPARTMENT OF ENERGY \\ under Contract DE-ACO5-76RL01830
}

Printed in the United States of America
Available to DOE and DOE contractors from the Office of Scientific and Technical Information, P.O. Box 62, Oak Ridge, TN 37831-0062; ph: (865) 576-8401 fax: (865) 5765728
email: reports@adonis.osti.gov




\section{Effect of Background Emissivity on Gas Detection in Thermal Hyperspectral Imagery}

SJ Walsh

MF Tardiff

LK Chilton

CN Metoyer

September 2008

Prepared for

the U.S. Department of Energy

Under Contract DE-AC05-76RL01830

Pacific Northwest National Laboratory

Richland, Washington 99352 


\section{Executive Summary}

Detecting and identifying weak gaseous plumes using thermal imaging data is complicated by many factors. These include variability due to atmosphere, ground and plume temperature, and background clutter. This paper presents an analysis of one formulation of the physics-based radiance model, which describes at-sensor observed radiance. The background emissivity and plume/ground temperatures are isolated, and their effects on chemical signal are described. This analysis shows that the plume's physical state, emission or absorption, is directly dependent on the background emissivity and the plume/ground temperatures. We then describe conditions on the background emissivity and plume/ground temperatures that have inhibiting or amplifying effects on the chemical signal. These claims are illustrated by analyzing synthetic hyperspectral imaging data with the Adaptive Matched Filter using four chemicals and three distinct background emissivities. Two chemicals (Carbontetrachloride and Tetraflourosilane) used in the analysis had a single dominant absorbance feature over a short range of wavenumbers. Analysis of simulated hyperspectral images containing these chemicals showed that, depending on the relationship between the plume and ground temperatures, the detectability of these gases over each of the backgrounds was either directly related or inversely related to the relative magnitude of the background emissivities. These empirical results are consistent with the analysis of the physics based model. The other chemicals considered (Ammonia and Tributylphosphate) exhibited more complex absorbance structure across the longwave infrared spectrum. Analysis of images containing these chemicals revealed that the the analysis of the physics-based model did not hold completely for these complex chemicals but did indicate that gas detection was dominated by their dominant absorbance features. These results provide a partial explanation of the effect of the background emissivity on gas detection. A more general exploration of gas absorbance/background emissivity variability and their effects on gas detection is warranted. 


\section{Contents}

1 Introduction ........................ 1

2 Physics-Based Radiance Model and Analysis . . . . . . . . . . 2

2.1 Case $1: T_{p}=T_{g} \ldots \ldots \ldots \ldots \ldots$

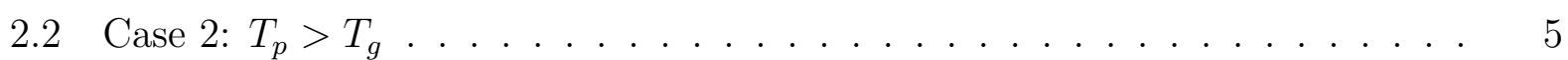

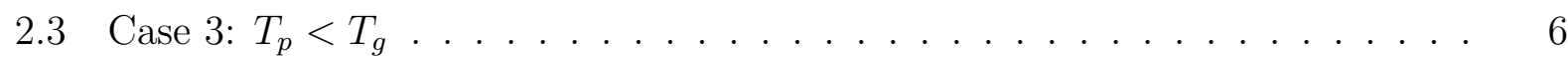

2.4 Graphical Summary of $T E$ for the three temperature cases. . . . . . . . . . . 6

3 Experimental Methods .................... 7

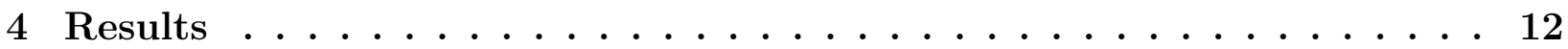

4.1 Single-feature Chemicals . . . . . . . . . . . . . . . . . . 12

4.2 Multi-feature Chemicals . . . . . . . . . . . . . . 13

5 Conclusions ........................ 21

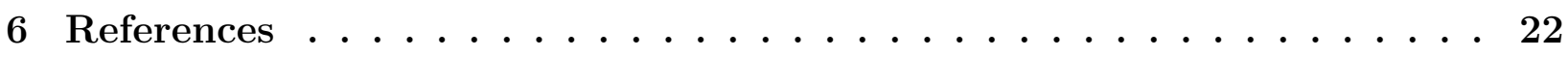




\section{List of Figures}

1 Temperature emissivity contrast plotted as a function of emissivity for three temperature cases at $\nu=1000 \mathrm{~cm}^{-1}: 305 K=T_{p}>T_{g}=300 K$ (red line), $T_{p}=T_{g}=300 K$ (green dashed line), and $295=T_{p}<T_{g}=300 K$ (orange line). $T E$ is non-negative when $T_{p} \geq T_{g}$, which indicates the plume is in emission. $T E$ can be positive or negative, depending on $\epsilon_{g}$ when $T_{p}<T_{g}$, which indicates the plume can be in emission or absorption. . . . . . . . .

2 (a) Background emissivity spectra used in image simulation. (b) Chemical Absorbance Spectra for $\mathrm{CCL}_{4}$ and $\mathrm{SiF}_{4}$. (c) Chemical Absorbance Spectra for $\mathrm{NH}_{3}$ and TBP. . . . . . . . . . . . . . . .

3 Detection proportions for $\mathrm{CCL}_{4}$ when (a) $T_{p}=T_{g}=300 \mathrm{~K}$, (b) $T_{p}=305 \mathrm{~K}>$ $T_{g}=300 K$, (c) $T_{p}=295 K<T_{g}=300 K$, and (d) gas absorbance spectra and background emissivities. . . . . . . . . . . . . .

4 Detection proportions for $\mathrm{SiF}_{4}$ when (a) $T_{p}=T_{g}=300 K$, (b) $T_{p}=305 K>$ $T_{g}=300 K$, (c) $T_{p}=292 K<T_{g}=300 K$, and (d) gas absorbance spectra and background emissivities. . . . . . . . . . . . . . . . . . 15

5 Background emissivities plotted with (a) $\mathrm{NH}_{3}$ and (b) TBP. . . . . . . . 16

6 Detection proportions for $\mathrm{NH}_{3}$ when (a) $T_{p}=T_{g}=300 K$, (b) $T_{p}=305 \mathrm{~K}>$ $T_{g}=300 K,(\mathrm{c}) T_{p}=295 K<T_{g}=300 K$, and $(\mathrm{d}) T_{p}=290 K<T_{g}=300 K$

7 Detection proportions for TBP when (a) $T_{p}=T_{g}=300 K$, (b) $T_{p}=305 K>$ $T_{g}=300 K,(\mathrm{c}) T_{p}=295 K<T_{g}=300 K$, and $(\mathrm{d}) T_{p}=290 K<T_{g}=300 K$. 


\section{Introduction}

Remotely detecting and identifying weak gaseous plumes using infrared measurement instruments is a challenge that receives continual attention. Burr and Hengartner [2] provide a comprehensive review of this problem. Generally, the ability to detect a gaseous effluent is influenced by its concentration path-length, atmospheric interferences, the temperature difference between the plume and the background surface, the emissivity of the background, and the complexity of the background surface. Collectively, these elements are termed clutter.

The type and variability of background clutter in an image presents many modeling challenges for gaseous plume detection. This subject has been studied from many perspectives. There have been studies that address the statistical characterization of background clutter at the pixel level of resolution [1,12]. End member estimation is a family of methods that models the types of clutter in an image on a subpixel (multiple background types within a pixel) level $[3,13]$. There are also studies that address signal-to-noise (SNR) and signal-toclutter (SCR) ratios $[11,4]$. The effects of signal contamination within a matched filter as well as methods to improve SCR are well studied.

The work presented in this paper studies background clutter from a different perspective. We develop a conceptual model of the effect of the background emissivity on the chemical signal as influenced by the temperature emissivity $(T E)$ contrast; that is, we identify when the background emissivity will have inhibiting effects on signal strength resulting in a reduced SCR. We view the temperature emissivity contrast as a difference in radiance signal contribution of the plume and the background. First, we isolate the $T E$ in a formulation of the physics-based radiance model. Then, we conduct analyses of the TE's contribution to the chemical signal while isolating the effect of the background emissivity. We describe the contributions of these terms to the chemical signal and identify cases where the difference between the plume and ground temperatures along with background emissivity have amplifying or inhibiting effects upon the net radiance signal at the sensor.

We validate our conceptual model for the effects of emissivity and temperature contrast on signal strength by simulating simplified hyperspectral images using the InfraRed Systems Analysis in General Environments (IR-SAGE) code [8] and calculating relative detection proportions for three different backgrounds. We investigate three temperature cases and four gases. The temperature cases consist of a constant ground temperature with plume 
temperatures that are less than, equal to, and greater than the ground temperature. We estimate relative probabilities of detection using an adaptive matched filter for gases that have a single large spectral feature. We also test the conceptual model further with two gases that have multiple dominant spectral features.

This paper is organized as follows. Section 2 presents the physics-based radiance model and the analyses of the temperature emissivity contrast cases that demonstrate the influence of background emissivity upon gas detection. Section 3 describes image simulation, the gas detection method, and sets up the modeling experiments with the four gases and three backgrounds to demonstrate the phenomena described in Section 2. Section 4 presents the results of the simulation studies and Section 5 presents our conclusions.

\section{Physics-Based Radiance Model and Analysis}

We explore the three-layer physics-based radiance model which relates ground, plume, and atmospheric radiance to gain insight into how the structure of the background emissivity impacts the at-sensor observed plume signal $[5,2,7]$. This model can be written as

$$
L_{o b s}(\nu)=\tau_{a}(\nu)\left[\left(1-\tau_{p}(\nu)\right) B\left(T_{p} ; \nu\right)+\tau_{p}(\nu) L_{g}(\nu)\right]+L_{u}(\nu)+e(\nu)
$$

where $L_{o b s}(\nu)$ represents sensor-recorded radiance in $W / \mathrm{cm}^{2} * S r * \mathrm{~cm}^{-1}$ at wavenumber $\nu\left(\mathrm{cm}^{-1}\right), \tau_{a}(\nu)$ and $\tau_{p}(\nu)$ are dimensionless terms representing the atmosphere and plume transmissivity, respectively, $B(T ; \nu)$ has radiance units and is Planck's Blackbody function at wavenumber $\nu$ and temperature $T(\mathrm{~K}), L_{g}(\nu)$ and $L_{u}(\nu)$ are the ground-leaving and atmospheric upwelling radiances, respectively, and $e(\nu)$ represents instrument noise and modeling error.

Following the convention of $[10,2]$, we model the ground-leaving radiance as

$$
L_{g}(\nu)=\epsilon_{g}(\nu) B\left(T_{g} ; \nu\right)
$$

where $\epsilon_{g}(\nu)$ is a dimensionless quantity representing the emissivity of the ground at wavenumber $\nu$, and $0 \leq \epsilon_{g}(\nu) \leq 1$. Note that this formulation ignores the reflected atmospheric downwelling radiance. This assumption is reasonable in the Longwave Infrared band (LWIR) 
because the reflected radiance contribution to observed signal is negligible [10].

The Beer-Bourger-Lambert Law [6] gives an explicit expression for the transmissivity of a gas in terms of the chemical effluent's concentration path-length, $c$ (with $c$ measured in parts-per-million-meter, denoted $p p m-m$ ), as follows:

$$
\tau_{p}(\nu)=e^{-A(\nu) c}
$$

where $A(\nu)$ is the absorbance coefficient of the gas in $p p m-m^{-1}[6]$. For optically thin plumes, this term is well approximated by the first two terms in a Taylor Series expansion [2]. This gives

$$
\tau_{p}(\nu) \approx 1-A(\nu) c
$$

We now substitute Eq. (2) and Eq. (4) into Eq. (1) to arrive at the working-gas-plume linear model

$$
L_{o b s}(\nu)=\tau_{a}(\nu)\left[B\left(T_{p} ; \nu\right)-\epsilon_{g}(\nu) B\left(T_{g} ; \nu\right)\right] A(\nu) c+\tau_{a}(\nu) \epsilon_{g}(\nu) B\left(T_{g}, \nu\right)+L_{u}(\nu)+e(\nu)
$$

which we interrogate further.

The right hand side of Eq. (5) shows that atmospheric radiance observed by the sensor is an additive layering of upwelling radiance $L_{u}(\nu)$, ground radiance attenuated by atmosphere $\tau_{a}(\nu) L_{g}(\nu)$, and the signal due to the chemical plume $\tau_{a}(\nu)\left[B\left(T_{p} ; \nu\right)-\epsilon_{g}(\nu) B\left(T_{g} ; \nu\right)\right] A(\nu) c$. This representation has been used to motivate scene whitening and the use of the AdaptiveMatched Filter $(A M F)$ for gas detection $[2,7]$. We will use this formulation to explore the effect that background emissivity and the ground/plume temperatures have on the chemical signal.

The radiance due to the chemical plume is the first term on the right hand side of Eq. (5), namely

$$
\tau_{a}(\nu)\left[B\left(T_{p} ; \nu\right)-\epsilon_{g}(\nu) B\left(T_{g} ; \nu\right)\right] A(\nu) c .
$$

Inspection of this term shows that radiance due to the plume is a function of atmospheric transmission, plume and ground temperature, ground emissivity, and the plume gas absorbance and concentration path length. We are interested in how the structure of the background emissivity affects the chemical signal. Toward that end, we will make the following assumptions: 
1. The gas exhibits absorbance at wavenumber $\nu$.

2. The gas is present at some fixed concentration path length $c$.

3. The atmospheric transmission, $\tau_{a}(\nu)$ is such that all plume radiance is not being attenuated by atmosphere at wavenumber $\nu$.

Assumptions 1 and 2 confirm that there is a chemical signal (gas absorbance or concentration path length of 0 imply chemical signal of 0 ). Assumption 3 confirms that chemical radiance can pass through the atmosphere and reach the sensor.

To isolate the effects of background emissivity on the net plume signal at a single channel, we will focus our attention on the Temperature Emissivity Contrast that is defined below

$$
T E\left(T_{p}, T_{g}, \epsilon_{g}, \nu\right)=B\left(T_{p} ; \nu\right)-\epsilon_{g}(\nu) B\left(T_{g} ; \nu\right)
$$

Eq. (6) shows that the chemical signal is proportional to $T E$. Thus, larger values of $T E$ (in absolute value) will yield larger chemical signals at wavenumber $\nu$, making the plume easier to detect at that wavenumber. Therefore, we investigate the properties of the background emissivity $\epsilon_{g}(\nu)$ that will yield a larger $T E$. We consider three plume-ground temperature cases: $T_{p}=T_{g}, T_{p}>T_{g}$, and $T_{p}<T_{g}$. We analyze $T E$ for each of these temperature cases in the next sections.

\section{$2.1 \quad$ Case 1: $T_{p}=T_{g}$}

This case is presented first since it is the easiest to interpret analytically. Equality of the plume and ground temperatures implies that $B\left(T_{p} ; \nu\right)=B\left(T_{g} ; \nu\right)=B(T ; \nu)$ for the common temperature $T$. Thus, when $T_{p}=T_{g}=T$, we can express Eq. (7) as

$$
\begin{aligned}
T E\left(T_{p}, T_{g}, \epsilon_{g}, \nu\right) & =B\left(T_{p} ; \nu\right)-\epsilon_{g}(\nu) B\left(T_{g} ; \nu\right) \\
& =B(T ; \nu)-\epsilon_{g}(\nu) B(T ; \nu) \\
& =\left(1-\epsilon_{g}(\nu)\right) B(T ; \nu)
\end{aligned}
$$


Since $0 \leq \epsilon_{g}(\nu) \leq 1$, Eq. (8) demonstrates that a smaller $\epsilon_{g}(\nu)$ will result in a larger $T E$ and hence a larger chemical signal. We also note that $T E$ is strictly positive for this temperature case. An $\epsilon_{g}(\nu)=1$ is required for $T E=0$, but this happens only for a true Blackbody. This indicates that the plume is in emission (emitting radiation) when $T_{p}=T_{g}$. A gas plume in emission is contributing to the observed radiance signal, and the chemical may be seen as peaks on the observed radiance at wavenumbers where the gas exhibits positive values in $A(\nu)$.

\subsection{Case 2: $T_{p}>T_{g}$}

When $T_{p}>T_{g}$, it follows that $B\left(T_{p} ; \nu\right)>B\left(T_{g} ; \nu\right)$ since Planck's function is monotonically increasing with respect to $T$. We can write the plume's Blackbody radiance as a function of the ground's blackbody radiance. We express this as

$$
B\left(T_{p} ; \nu\right)=\left(1+\delta_{1}(\nu)\right) B\left(T_{g} ; \nu\right)
$$

where we use $\delta_{1}(\nu)$ to represent the relative difference between the plume and ground Blackbody functions as follows:

$$
\delta_{1}(\nu)=\frac{B\left(T_{p} ; \nu\right)-B\left(T_{g} ; \nu\right)}{B\left(T_{g} ; \nu\right)} .
$$

Substituting Eq. (9) into Eq. (7) yields

$$
\begin{aligned}
T E\left(T_{p}, T_{g}, \epsilon_{g}, \nu\right) & =B\left(T_{p} ; \nu\right)-\epsilon_{g}(\nu) B\left(T_{g} ; \nu\right) \\
& =\left(1+\delta_{1}(\nu)\right) B\left(T_{g} ; \nu\right)-\epsilon_{g}(\nu) B\left(T_{g} ; \nu\right) \\
& =\left(1+\delta_{1}(\nu)-\epsilon_{g}(\nu)\right) B\left(T_{g} ; \nu\right) .
\end{aligned}
$$

Eq. (11) is also easy to interpret as a function of $\epsilon_{g}$. First, we note that because $\delta_{1}(\nu)$ is a positive and is additive in $T E$, it can be shown that $T E$ is strictly positive for this temperature case. This says that the plume is strictly in emission for this case. It also says that a larger chemical signal is observable compared to the previous temperature case. Since $\epsilon_{g}(\nu)$ is subtracted, small emissivities yield a larger chemical signal at wavenumber $\nu$. 


\subsection{Case 3: $T_{p}<T_{g}$}

This temperature case is the most difficult to interpret analytically. When $T_{p}<T_{g}$, it follows that $B\left(T_{p} ; \nu\right)<B\left(T_{g} ; \nu\right)$. Similar to the previous section, we can write

$$
B\left(T_{p} ; \nu\right)=\left(1-\delta_{2}(\nu)\right) B\left(T_{g} ; \nu\right)
$$

where the $\delta_{2}(\nu)$ is now expressed as

$$
\delta_{2}(\nu)=\frac{B\left(T_{g} ; \nu\right)-B\left(T_{p} ; \nu\right)}{B\left(T_{g} ; \nu\right)}
$$

Note that the order of the Blackbody functions in Eq. (13) has changed from Eq. (10) to maintain a positive $\delta_{2}(\nu)$ function for this temperature case.

We substitute Eq. (12) into Eq. (7) and find that

$$
\begin{aligned}
T E\left(T_{p}, T_{g}, \epsilon_{g}, \nu\right) & =B\left(T_{p} ; \nu\right)-\epsilon_{g}(\nu) B\left(T_{g} ; \nu\right) \\
& =\left(1-\delta_{2}(\nu)\right) B\left(T_{g} ; \nu\right)-\epsilon_{g}(\nu) B\left(T_{g} ; \nu\right) \\
& =\left(1-\delta_{2}(\nu)-\epsilon_{g}(\nu)\right) B\left(T_{g} ; \nu\right) .
\end{aligned}
$$

The fact that $\delta_{2}(\nu)$ is a subtracted term in Eq. (14) complicates the interpretation of what properties of $\epsilon_{g}(\nu)$ are desirable for a larger $T E$ and larger chemical signal. We provide a graphical summary of $T E$ for the three temperature cases in the next subsection to clarify how $\epsilon_{g}(\nu)$ contributes to $T E$ for each temperature case. For this temperature case, TE can be positive, negative, or 0 , and this is directly dependent on $\epsilon_{g}(\nu)$ as well as $T_{p}$ and $T_{g}$. A plume in absorption $(T E<0)$ is decreasing the observed radiance signal at wavenumbers where $A(\nu)$ is positive. In this case, the gas can be seen as troughs in the observed radiance. A plume that is neither emitting nor absorbing $(T E=0)$ is transparent to the sensor and cannot be detected.

\subsection{Graphical Summary of $T E$ for the three temperature cases.}

We present a plot of $T E$ as a function of $\epsilon_{g}(\nu)$ for the three temperature cases at $\nu=1000$ $\mathrm{cm}^{-1}$ in Figure 1. We set the ground temperature to $T_{g}=300 \mathrm{~K}$ and vary the plume 
temperature at $T_{p}=305,300,295 K$.

The plots of $T E$ for the $T_{p}>T_{g}$ and $T_{p}=T_{g}$ cases are the red and green lines, respectively. The plot shows that values of $\epsilon_{g}(\nu)$ closer to 0 increase the magnitude of $T E$. This is consistent with the interpretation of the analyses.

The plot of $T E$ when $T_{p}<T_{g}$ illustrates which values of $\epsilon_{g}(\nu)$ yield a larger $T E$ in magnitude. We can see that $T E$ crosses the horizontal axis if $\epsilon_{g}(\nu)=B\left(T_{p} ; \nu\right) / B\left(T_{g} ; \nu\right)$. This shows that when $\epsilon_{g}(\nu)=B\left(T_{p} ; \nu\right) / B\left(T_{g} ; \nu\right)$, the plume is neither emitting nor absorbing and there is no chemical signal at wavenumber $\nu$. The fact that $T E$ can cross the horizontal axis informs us that small emissivities (closer to 0) or larger emissivities (closer to 1) have the potential to make the absolute value of $T E$ larger for this temperature case.

The implications of this plot are as follows. When the gas exhibits an absorbance feature at wavenumber $\nu$ and the plume is in emission, the analysis implies that backgrounds that have emissivities closer to 0 at wavenumber $\nu$ will contribute to a larger chemical signal, and thus the plume will be easier to detect. We note that this is true for each temperature case $T_{p} \geq T_{g}$ and $T_{p}<T_{g}$. However, when $T_{p}<T_{g}$, we observe that it is possible that a background emissivity closer to 0 or closer to 1 may contribute to a larger chemical signal. This is illustrated by the orange line in Figure 1. Thus the plume will give some chemical signal for emissivities near 1 when $T_{p}<T_{g}$ and this distinguishes this temperature case from those where $T_{p} \geq T_{g}$.

\section{Experimental Methods}

The goal of this section is to explore the validity of the analysis presented in Section 2 . While the analysis describes the effect of $\epsilon_{g}(\nu)$ on the chemical signal at a single channel, hyperspectral instruments record a radiance vector across the LWIR band. As such we will explore how these phenomena affect gas detectability in a multivariate setting. In order to illustrate the claims made in the single channel analysis, we will first restrict ourselves to a specific subset of gases and background emissivities. We choose gases that exhibit strong absorbance over a small range of wavenumbers and no absorbance everywhere else and select emissivities that do not change ordering over the wavenumbers where the gas exhibits absorbance. Then we explore the effect of background emissivities on detectibility 


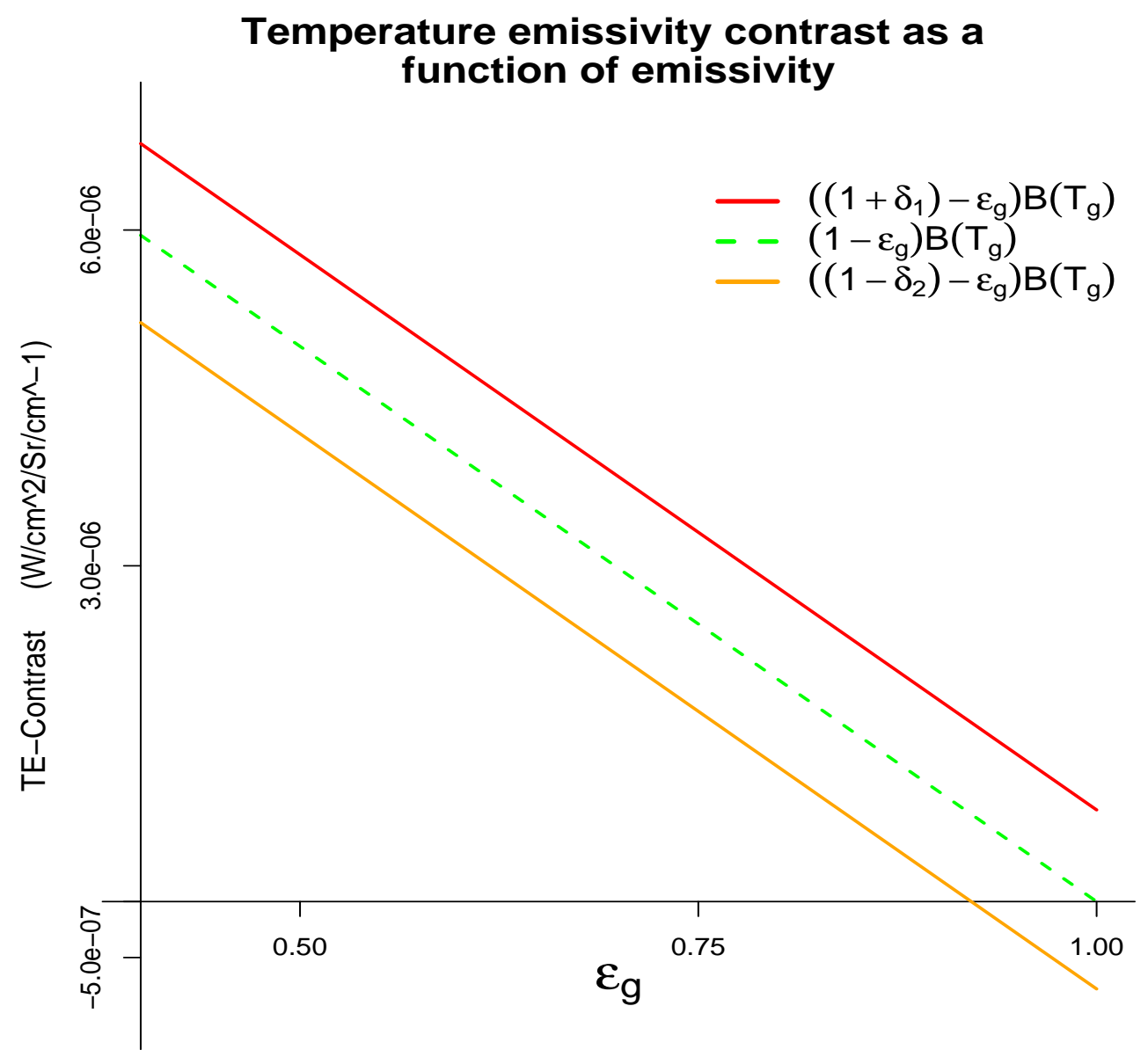

Figure 1: Temperature emissivity contrast plotted as a function of emissivity for three temperature cases at $\nu=1000 \mathrm{~cm}^{-1}: 305 \mathrm{~K}=T_{p}>T_{g}=300 \mathrm{~K}$ (red line), $T_{p}=T_{g}=300 \mathrm{~K}$ (green dashed line), and $295=T_{p}<T_{g}=300 K$ (orange line). $T E$ is non-negative when $T_{p} \geq T_{g}$, which indicates the plume is in emission. $T E$ can be positive or negative, depending on $\epsilon_{g}$ when $T_{p}<T_{g}$, which indicates the plume can be in emission or absorption.

using gases that do not meet this constraining criterion. We choose gases that have multiple features across all wavenumbers in the LWIR and do not require any specific ordering of the background emissivities where the gases exhibit features.

We employ IR-SAGE to simulate simplified hyperspectral images. The background spectra used in this study are laboratory-measured individual background materials from the Nonconventional Exploitation Factors Data System (NEFDS), a government database of surface reflection parameters. We selected three distinct background emissivity clusters and used the mean spectra of the three clusters in image simulation. These spectra are representative of the following three groups: Brick, Snow, and Steel-Copper Tubing. These spectra are 
presented in Figure 2(a). Note that in the LWIR $\left(750-1250 \mathrm{~cm}^{-1}\right)$, these emissivities are relatively high.

Two single feature gases were selected for image simulation: Carbontetrachloride $\left(\mathrm{CCL}_{4}\right)$ and Tetraflourosilane $\left(\mathrm{SiF}_{4}\right)$. These gases exhibit one large dominant absorbance feature. A plot of the absorbance spectra for these gases is presented in Figure 2(b). The spectrum for $\mathrm{CCL}_{4}$ shows a major feature around $790 \mathrm{~cm}^{-1}$. $\mathrm{SiF}_{4}$ exhibits a dominant feature around $1025 \mathrm{~cm}^{-1}$. Note that the background emissivities show a consistent ordering across type (they do not cross) where each of these chemicals exhibits positive absorbance.

We also selected two gases that exhibit multiple features across the spectrum. These gases are Ammonia $\left(\mathrm{NH}_{3}\right)$ and Tributylphosphate (TBP). A plot of the absorbance spectra for these gases is presented in Figure 2(c). The spectrum for $\mathrm{NH}_{3}$ exhibits multiple small and sharp peaks across many of the wavenumbers. The spectrum for $\mathrm{NH}_{3}$ shows two dominant sharp peaks at approximately 930 and $960 \mathrm{~cm}^{-1}$ and multiple minor sharp peaks across the other wavenumbers. The spectrum for TBP shows some absorbance (greater than 0) at all wavenumbers as well as some large broad peaks. The largest is centered at approximately $1050 \mathrm{~cm}^{-1}$ and covers a range from approximately 1040 to $1100 \mathrm{~cm}^{-1}$. We note that the background emissivites show no consistent ordering where these gases exhibit non-zero absorbance.

The simulated images have dimensions $75 \times 120 \times 126$ (rows by columns by spectral dimension). The wavenumber range used is 750 to $1250 \mathrm{~cm}^{-1}$ in steps of 4 . The three background spectra are inserted across the rows in three 25-pixel swaths. The chemicals are inserted as six 20-column bands at concentration path-lengths 16, 8, 4, 2, 1, and 0 ppm-m. This orientation produces 500 pixel replicates within a background/gas concentration combination.

Images were created for each temperature case. The ground temperature, $T_{g}$, was kept constant at $300 \mathrm{~K}$, and the plume temperature, $T_{p}$, was varied at $T_{p}=305 \mathrm{~K}, 300 \mathrm{~K}$, and $295 \mathrm{~K}$ for the $\mathrm{CCL}_{4}$ images. This temperature range was selected to illustrate the effect of the background emissivities on detection. A slightly wider temperature range was needed to illustrate the effect for $\mathrm{SiF}_{4}: T_{g}=300$ and $T_{p}=305 K, 300 K, 292 K$. It was possible to show the effect of background emissivity on gas detection for $\mathrm{NH}_{3}$ and TBP, but a larger temperature range was required. For these chemicals $T_{g}$ was kept constant at $300 K$ and $T_{p}$ was varied at $T_{p}=290 K, 295 K, 300 K$, and $305 K$. 


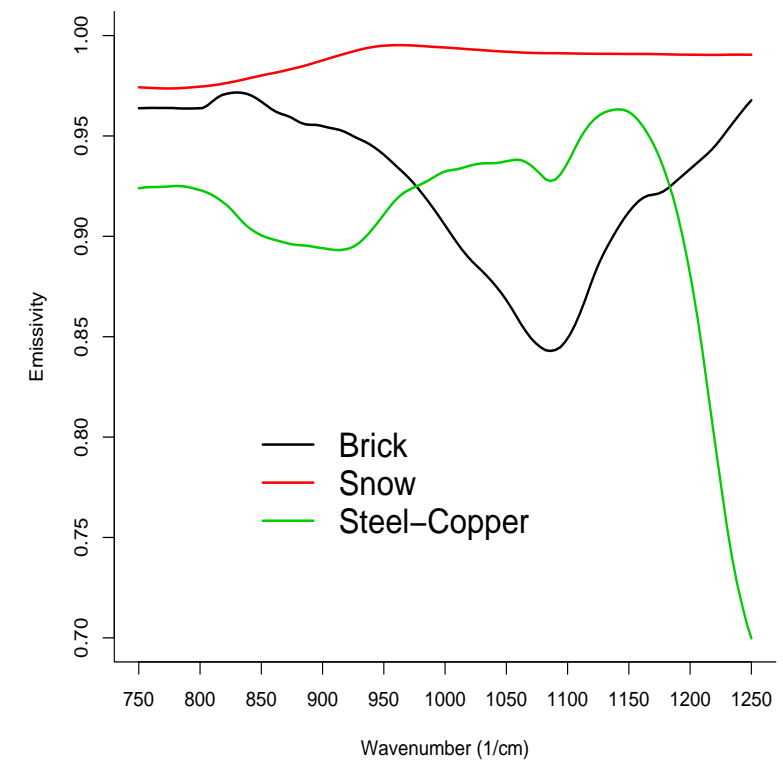

(a)

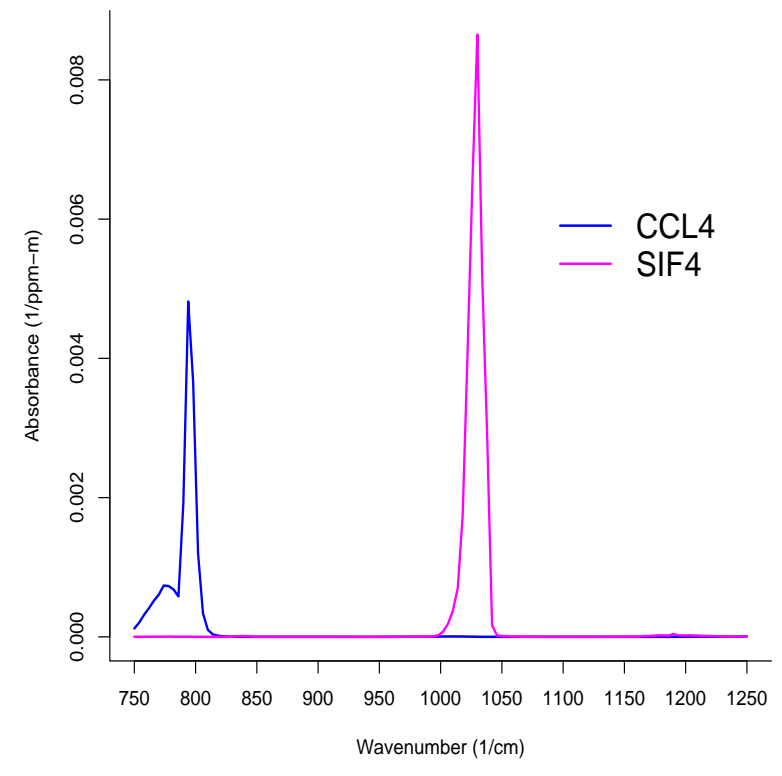

(b)

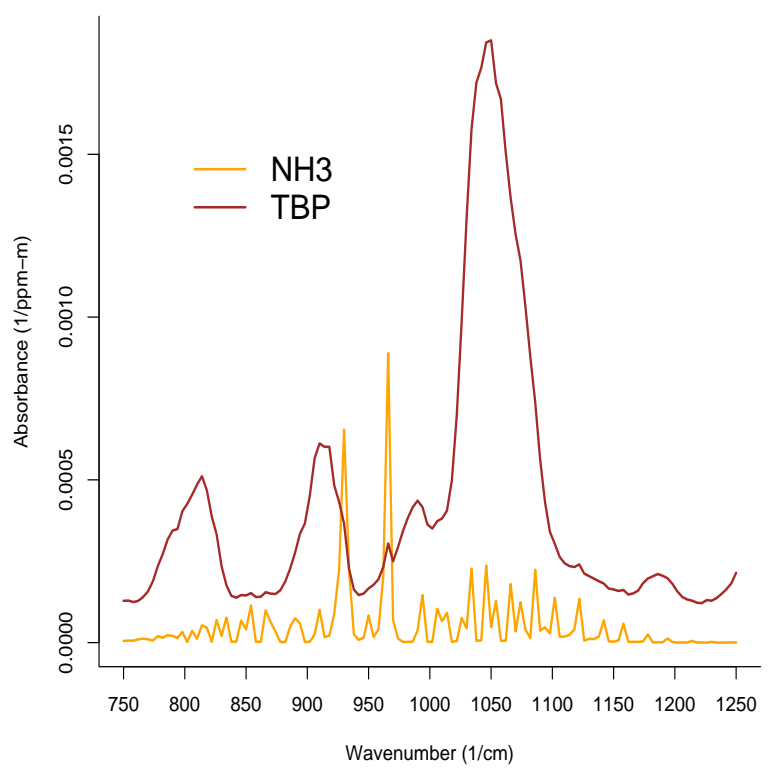

(c)

Figure 2: (a) Background emissivity spectra used in image simulation. (b) Chemical Absorbance Spectra for $\mathrm{CCL}_{4}$ and $\mathrm{SiF}_{4}$. (c) Chemical Absorbance Spectra for $\mathrm{NH}_{3}$ and TBP. 
Simulated zero-mean instrument noise was used to perturb the spectra in each pixel. Variability due to atmosphere, temperature, and emissivity from pixel to pixel were held constant to enable us to study the effect of the background emissivity's variability across the spectrum on the chemical signal.

We use the Adaptive Matched Filter $(A M F)$ as a gas detector, note that this is equivalent to a generalized least squares solution to a linear model e.g. see $[2,9]$. The image analysis process is as follows. The non-gas pixels can be formulated as

$$
\boldsymbol{L}_{o f f_{i}}=\boldsymbol{\tau}_{a} \odot \boldsymbol{L}_{g}+\boldsymbol{L}_{u}+\boldsymbol{e}_{i}, \quad i=1, \ldots, 500
$$

where bold terms are vectors of length 126 (126 spectral channels) and $\odot$ denotes the Hadamard product (elementwise multiplication). We compute the mean of these pixels for use in background radiance subtraction. This can be represented as $\overline{\boldsymbol{L}_{\text {off }}}=\boldsymbol{\tau}_{a} \odot \boldsymbol{L}_{g}+\boldsymbol{L}_{u}+\overline{\boldsymbol{e}}$ for the 500 non-gas replicates as the atmospheric transmissivity and background radiance are not varied across pixels. We subtract this quantity from each of the gas pixels that contain the same background type, i.e., we compute

$$
\boldsymbol{L}_{o b s}-\overline{\boldsymbol{L}_{o f f}}=\boldsymbol{\tau}_{a} \odot\left[\boldsymbol{B}\left(T_{p}\right)-\boldsymbol{\epsilon}_{g} \odot \boldsymbol{B}\left(T_{g}\right)\right] \odot \boldsymbol{A} c+\boldsymbol{e} .
$$

Eq. (16) shows how the background mean subtraction removes radiance due to ground as well as atmospheric upwelling radiance and leaves the chemical signal and noise. These data are processed with the $A M F$. Explicitly we compute

$$
A M F=\left(\boldsymbol{A}^{\prime} \hat{\boldsymbol{\Sigma}}^{-1} \boldsymbol{A}\right)^{-1} \boldsymbol{A}^{\prime} \hat{\boldsymbol{\Sigma}}^{-1}\left(\boldsymbol{L}_{o b s}-\overline{\boldsymbol{L}_{o f f}}\right)
$$

where $\boldsymbol{A}$ is a $126 \times 1$ vector of the gas absorbance spectra and $\hat{\boldsymbol{\Sigma}}: 126 \times 126$ represents the spectral covariance matrix computed on the non-gas pixels. This formulation of the filter is sometimes used in practice in the LWIR. It assumes no information about the atmosphere, plume or ground temperatures, and emissivity is available. It also assumes that $T E$ is constant across the spectral dimension $[2,1]$.

If the $A M F$ is statistically significant based on a $5 \%$ level two-sided hypothesis test [8], then we say we have "detected" the gas in that pixel. We apply this solution to each of the 500 replicates within each gas concentration path-length/background combination and then 
record the proportion of detections:

$$
\hat{p}=\frac{\# \text { of detections }}{500}
$$

We use $\hat{p}$ as an estimate of the gas detection probability. We get 18 detection probabilities for each image as there are three backgrounds and six gas-concentration path-length levels. Lastly, we plot detection proportions versus concentration path length and look for orderings (conditioned on background emissivity) in these curves. We then interpret these results back to the claims to the effect that background emissivities should produce a larger chemical signal and better detectability for each chemical.

\section{Results}

In this section, we present the results of analyses performed on the synthetic IR-SAGE images. Sample detection proportions were computed for each combination of concentration path-length and background type. The detection proportions for the single-feature chemicals, $\mathrm{CCL}_{4}$ and $\mathrm{SiF}_{4}$, are presented in Figures 3 and 4 respectively. The detection proportions for the multi-feature chemicals, $\mathrm{NH}_{3}$ and TBP, are presented in Figures 6 and 7 respectively. The single and multi-feature chemicals are considered in separate sections below.

\subsection{Single-feature Chemicals}

First, we consider Figure 3(a), which is a plot of the empirical detection curves for $\mathrm{CCL}_{4}$ when $T_{p}=T_{g}=300 \mathrm{~K}$. Examination of the plot shows that the backgrounds can be ordered by detection proportion as (best to worst) Steel-Copper, Brick, and Snow. Figure 3(d) shows a plot of the $\mathrm{CCL}_{4}$ absorbance spectra along with the background emissivities. We see that where $\mathrm{CCL}_{4}$ exhibits the large absorbance peak, the background emissivities can be ordered from low to high as Steel-Copper, Brick, and Snow. Thus, the results here are consistent with the characterization of $T E$ for this temperature case: lower background emissivities contribute to larger chemical signal when the plume is in emission.

Next, we consider the detection proportion plot for $305 \mathrm{~K}=T_{p}>T_{g}=300 \mathrm{~K}$ in Figure 3(b) which shows the same ordering in backgrounds as the previous temperature case. It also 
shows that detection has generally increased over all backgrounds. These observations are consistent with the characterization of $T E$ as they represent the fact that the backgrounds that give better detection for the $T_{p}=T_{g}$ case are the same as for the $T_{p}>T_{g}$ case. They also represent the fact that $T_{p}>T_{g}$ gives a larger $T E$ and in turn a larger chemical signal.

Third, we consider Figure 3(c), which shows the empirical detection curves for $\mathrm{CCL}_{4}$ when $295 K=T_{p}<T_{g}=300 K$. Now we see that the ordering in the detection curves by background is Snow, Brick, and then Steel Copper. Inspection of Figure 3(d) shows that the background emissivites can be ordered (high to low) as Snow, Brick, then Steel-Copper where $\mathrm{CCL}_{4}$ exhibits its dominant absorbance peak. These results are consistent with the analysis of $T E$ for this temperature case. They illustrate that, for an absorbing plume and a large enough (negative) $T E$, the backgrounds that give best detection are those that exhibit larger emissivities and not smaller ones as in the previous two temperature cases.

Now we consider Figure 4(a), which is a plot of the empirical detection curves for $\mathrm{SiF}_{4}$ when $T_{p}=T_{g}=300 \mathrm{~K}$. Examination of the plot shows that the backgrounds can be ordered from best to worst detectability for $\mathrm{SiF}_{4}$ as Brick, Steel-Copper, and Snow. Figure 4(d) shows that the background emissivities can be ordered from least to greatest as Brick, Steel-Copper, and Snow where $\mathrm{SiF}_{4}$ exhibits its dominant absorbance peak. This ordering is consistent with the analysis of $T E$ : smaller emissivities yield a larger chemical signal for this case.

Next, we consider Figure 4(b), which gives the $\mathrm{SiF}_{4}$ detection proportions when $305 K=$ $T_{p}>T_{g}=300 K$. Examination of this plot shows that the background orderings by detection proportion are the same as the $T_{p}=T_{g}$ case. The plot also shows that detection has generally increased for the emitting plume at a higher temperature.

Last, we consider the plot in Figure 4(c), which presents the empirical detection proportions when $T_{p}=292 \mathrm{~K}<T_{g}=300 \mathrm{~K}$. This plot shows that the backgrounds that yield best detection have changed ordering to Snow, Steel-Copper, and Brick. Again, we see that larger emissivities yield larger detection proportions for a large $T E$ when $T_{p}<T_{g}$.

\subsection{Multi-feature Chemicals}

First, we present plots of the background emissivities and $\mathrm{NH}_{3}$ and TBP in Figure 5. The plot of the background emissivities with the $\mathrm{NH}_{3}$ spectrum in Figure 5(a) shows that the 


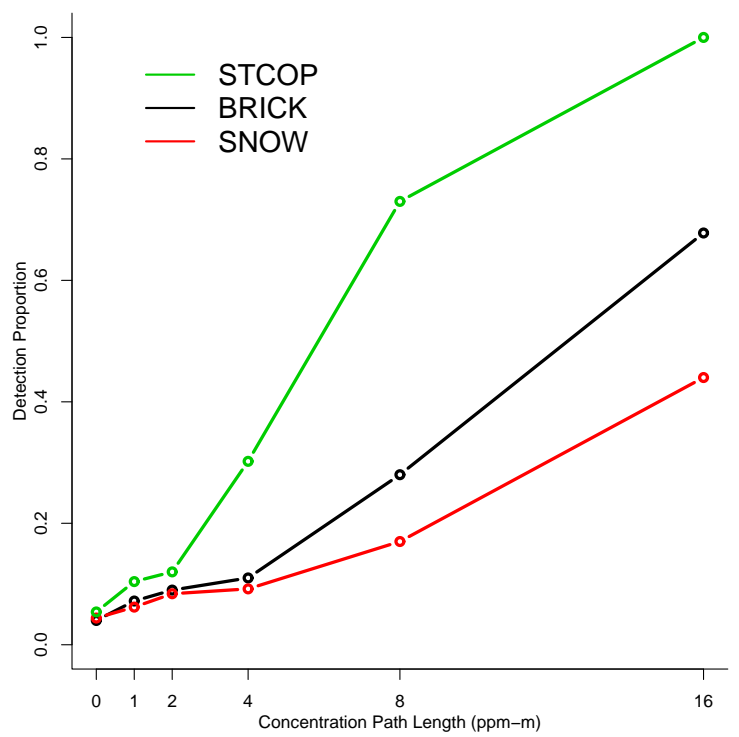

(a)

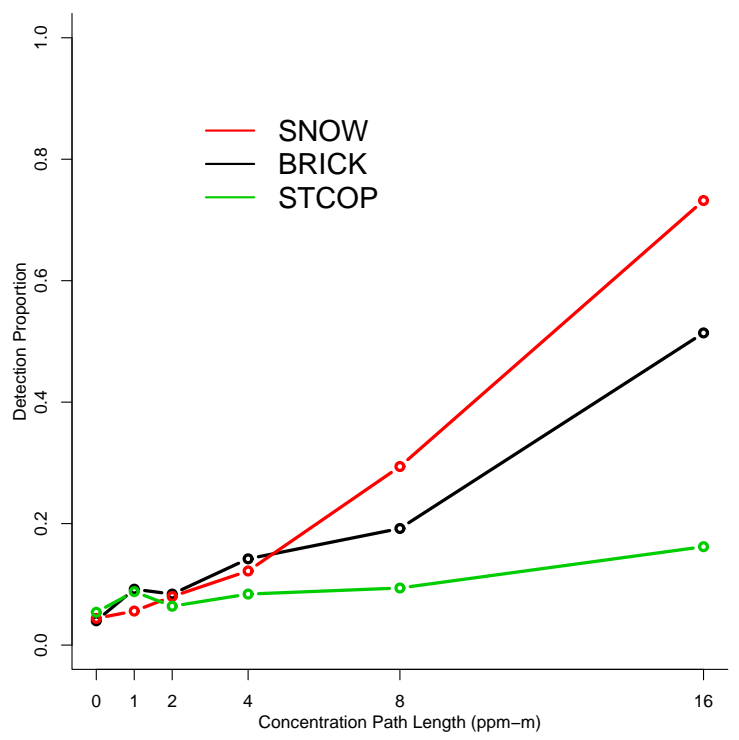

(c)

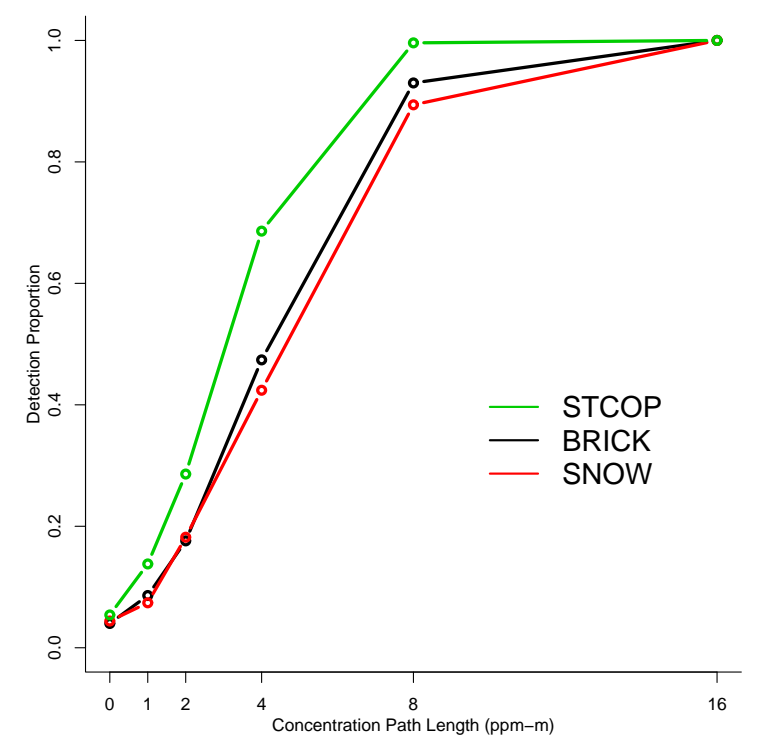

(b)

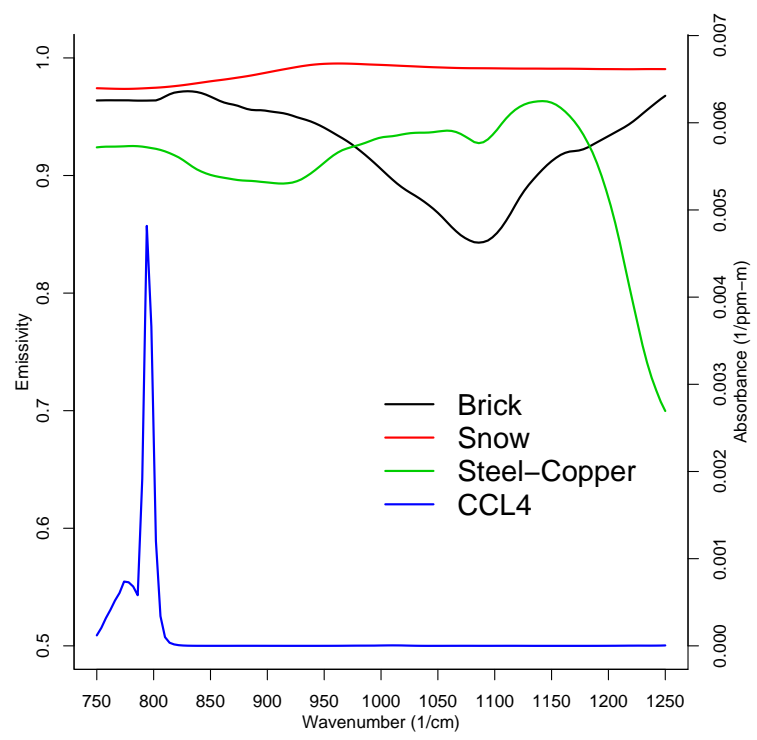

(d)

Figure 3: Detection proportions for $\mathrm{CCL}_{4}$ when (a) $T_{p}=T_{g}=300 K$, (b) $T_{p}=305 K>$ $T_{g}=300 K$, (c) $T_{p}=295 K<T_{g}=300 K$, and (d) gas absorbance spectra and background emissivities. 


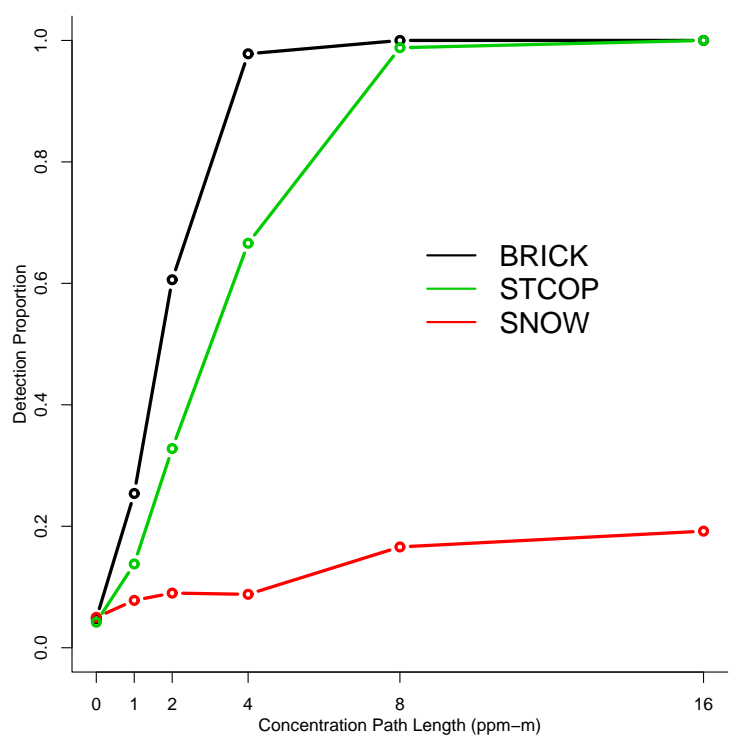

(a)

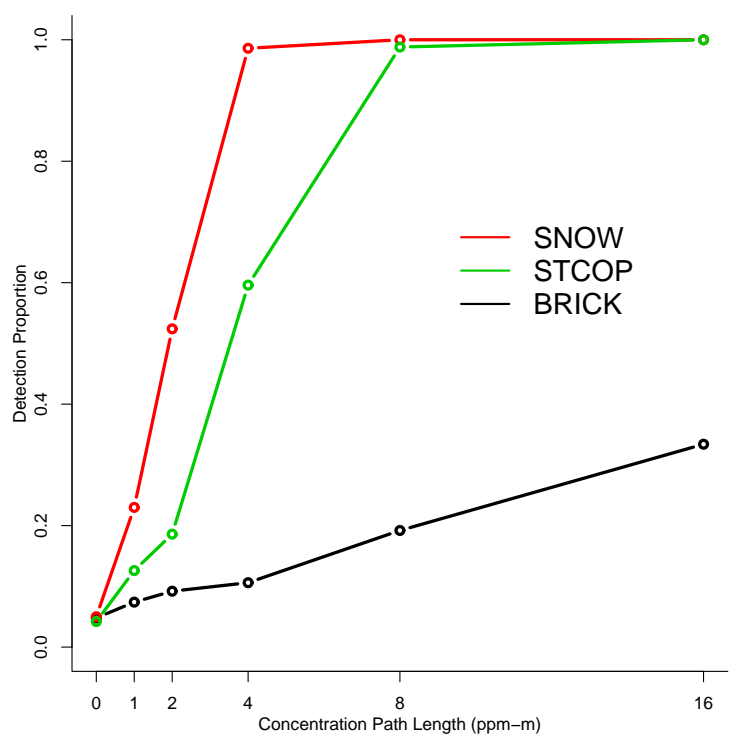

(c)

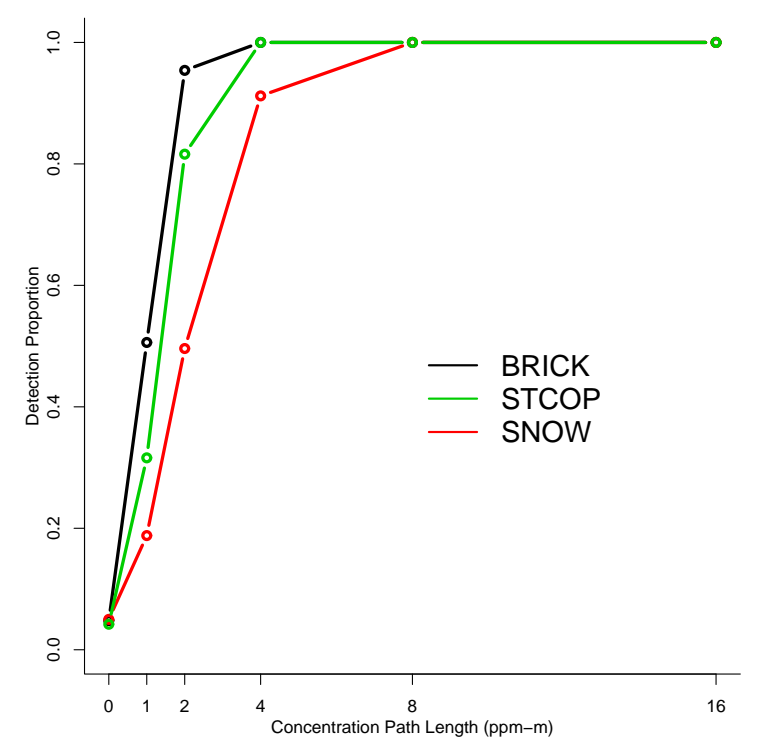

(b)

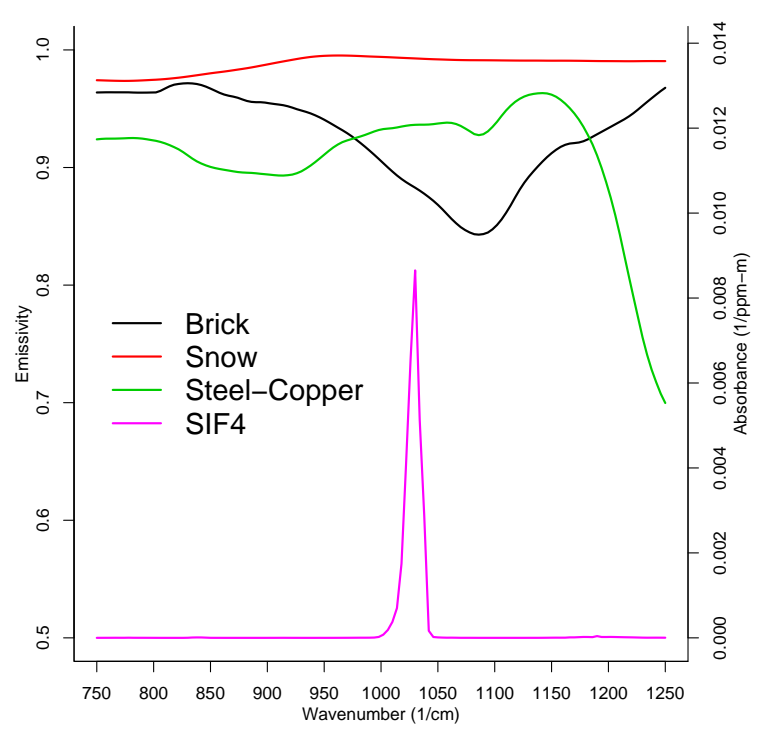

(d)

Figure 4: Detection proportions for $\mathrm{SiF}_{4}$ when (a) $T_{p}=T_{g}=300 K$, (b) $T_{p}=305 K>$ $T_{g}=300 K$, (c) $T_{p}=292 K<T_{g}=300 K$, and (d) gas absorbance spectra and background emissivities. 
background emissivities do not exhibit any consistent ordering across the wavenumbers where $\mathrm{NH}_{3}$ has non-zero absorbance peaks. While this is the case, we note that $\mathrm{NH}_{3}$ exhibits two dominant absorbance peaks at approximately 930 and $960 \mathrm{~cm}^{-1}$ and that, at these wavenumbers, it is possible to order the background emissivities as (high to low) Snow, Brick, then Steel-Copper. This point will be revisited in the presentation of the detection results. Similarly Figure 5(b) shows that the background emissivities show no consistent ordering across the wavenumbers where TBP exhibits non-zero absorbance features. We do note that TBP exhibits a broad dominant absorbance centered at approximately $1050 \mathrm{~cm}^{-1}$. At this wavenumber the background emissivities do show a consistent ordering as Snow, Steel-Copper, and Brick.

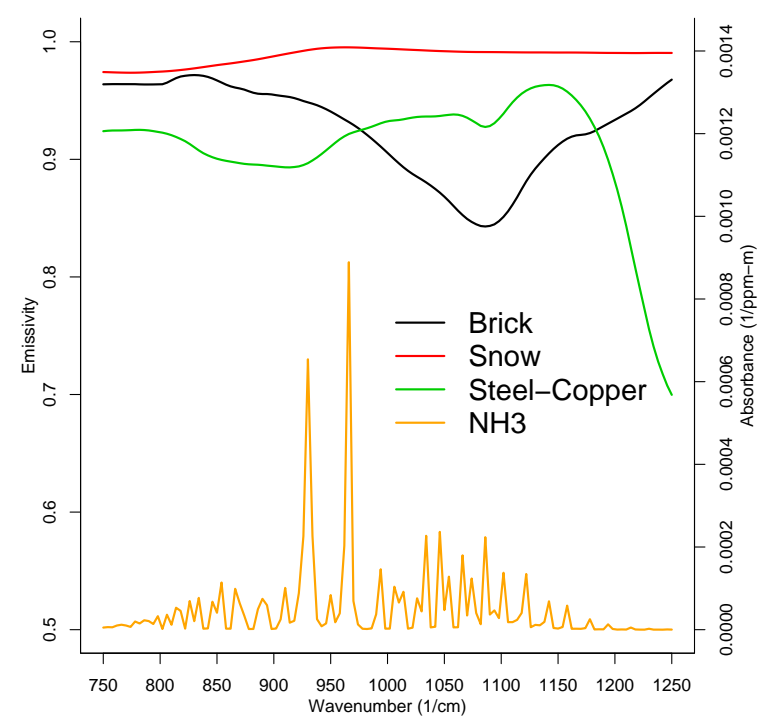

(a)

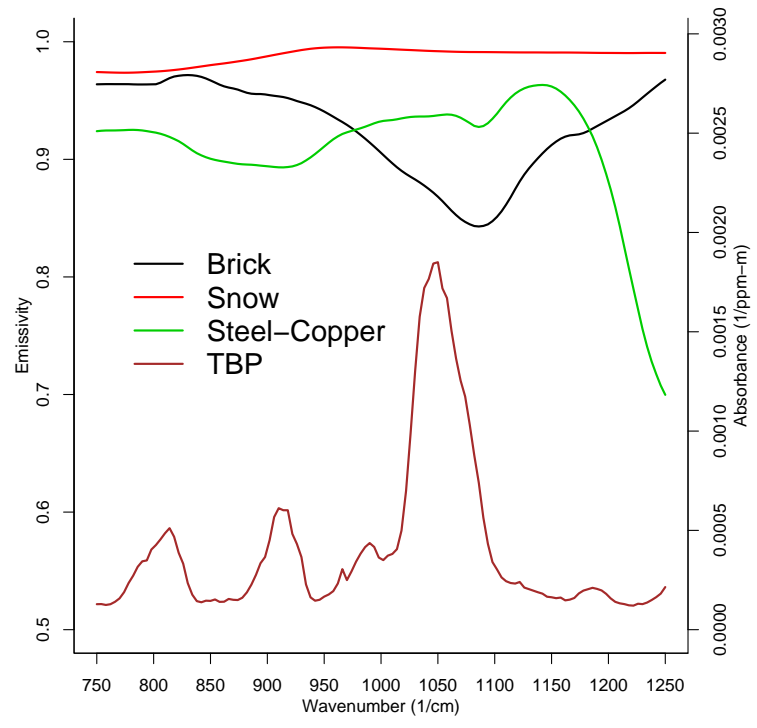

(b)

Figure 5: Background emissivities plotted with (a) $\mathrm{NH}_{3}$ and (b) TBP.

The results for $\mathrm{NH}_{3}$ and TBP presented in Figures 6 and 7 respectively indicate that it is possible to observe a switch in background orderings for best detection as the plume temperature goes from $T_{p}>T_{g}$ to $T_{p}<T_{g}$. The analysis of the physics based model in Section 2, however, does not lend to predicting the best/worst backgrounds for detection for these chemicals as they exhibit complex structure and relationship to each of the backgrounds across the spectrum.

First, we consider Figure 6(a) which presents the empirical detection results for $\mathrm{NH}_{3}$ when $T_{p}=T_{g}=300 K$. Examination of this plot shows that the backgrounds can be ordered by 
detection proportion as (best to worst) as Steel-Copper, Brick, then Snow. We note that the detection proportions for this temperature case are relatively low at each gas concentration path-length. Reasonably large detections are not observed until $16 \mathrm{ppm}-\mathrm{m}$. Further the detection proportion curve for $\mathrm{NH}_{3}$ over Snow is not statistically different from non-detection at any concentration path-length. We do note that at 930 and $960 \mathrm{~cm}^{-1}$ where $\mathrm{NH}_{3}$ exhibits the two dominant absorbance peaks, the backgrounds can be ordered (low to high) as SteelCopper, Brick, then Snow and that this is consistent with the detection proportion ordering at $16 \mathrm{ppm}-\mathrm{m}$. This observation suggests that the two large absorbance peaks for $\mathrm{NH}_{3}$ have the most impact on gas detection for these data. We also note that these observations are also consistent with the analysis of the physics-based model in Section 2 that when $T_{p}=T_{g}$ smaller emissivities yield a larger chemical signal for this case.

Second, we consider the detection proportion plot for $T_{p}=305 \mathrm{~K}>T_{g}=300 \mathrm{~K}$ in Figure 6(b). As expected, this plot shows that background ordering is the same as the previous temperature case at $16 \mathrm{ppm}-\mathrm{m}$ and that detection proportion has generally increased. This is consistent with with the characterization of $T E$ in Section 2 that larger temperature differences contribute to larger chemical signal.

Third, we consider the detection proportion plot for $295 \mathrm{~K}=T_{p}<T_{g}=300 \mathrm{~K}$ in Figure 6(c). This plot indicates that Snow is now the best background for detecting $\mathrm{NH}_{3}$ which is in contrast to the previous temperature cases. It also shows that the detection proportion curves for Steel-Copper and Brick have not yet changed ordering as we expect and in fact, these detection proportions are not significantly different from non-detection. This indicates that this temperature contrast is not sufficient to produce a $T E$ large enough to detect $\mathrm{NH}_{3}$ over these backgrounds. Recall that in this temperature case it is possible for $T E$ to change sign from positive to negative (see Section 2). This fact may help explain why $\mathrm{NH}_{3}$ cannot be detected over Steel-Copper and Brick, that is, these temperatures yield a $T E$ that gives a very small chemical signal.

Last, we consider the detection proportion plot for $290 \mathrm{~K}=T_{p}<T_{g}=300 \mathrm{~K}$ in Figure 6(c). This plot shows that the backgrounds can now be ordered as Snow, Brick, then Steel-Copper and this is exactly the opposite ordering from the $T_{p}=T_{g}$ and $T_{p}>T_{g}$ cases. We note that at 930 and $960 \mathrm{~cm}^{-1}$ where $\mathrm{NH}_{3}$ exhibits the two dominant absorbance peaks the background emissivities can be ordered (high to low) as Snow, Brick, then Steel-Copper. Again, these observations suggest that the large absorbance peaks of $\mathrm{NH}_{3}$ have the greatest impact on 
gas detection. We also note that the ordering of backgrounds for this temperature case are consistent with the analysis of $T E$ in Section 2 that when $T_{p}<T_{g}$ larger emissivities yield a larger chemical signal.

We now consider the detection results for TBP. We first consider the detection proportion plot for $T_{p}=T_{g}=300 \mathrm{~K}$ presented in Figure $7(\mathrm{a})$. The plot shows that the backgrounds can be ordered for best to worst detectability for TBP as Brick, Steel-Copper, and Snow. We note that TBP detections over the snow background were not statistically different from non-detections for this temperature case. While TBP exhibits complex structure and relationship to the background emissivities, we do note that at $1050 \mathrm{~cm}^{-1} \mathrm{TBP}$ exhibits its largest absorbance feature and that the backgrounds can be ordered as (low to high) Brick, Steel-Copper, and Snow. This ordering is consistent with the ordering of the detection proportion curves for this temperature case. These observations suggest that, while TBP exhibits non-zero absorbance over all wavenumbers in the LWIR, its detectability seems to be dominated by its largest absorbance feature. We also note that the ordering of the backround emissivities over the dominant absorbance feature is consistent with the analysis of $T E$ in Section 2 that when $T_{p}=T_{g}$, smaller background emissivities yield a larger chemical signal.

Second, we consider the detection proportion plot for $305 K=T_{p}>T_{g}=300 K$ presented in Figure $7(\mathrm{~b})$. We observe that the detection proportions have generally increased for this temperature case and that the backgrounds that give the best to worst detection for TBP are ordered the same as the previous temperature case at $16 \mathrm{ppm}-\mathrm{m}$. These observations are consistent with the analysis of $T E$ in Section 2 and show that a stronger chemical signal is observable with a greater plume-ground temperature difference.

Third, we consider the detection proportion plot for $295=T_{p}<T_{g}=300 \mathrm{~K}$ presented in Figure 7(c). We observe that, for this temperature case, the backgrounds can be ordered for best to worst detection for TBP as Snow, Brick, then Steel-Copper. These observations show that this temperature case is not yet sufficient to give a large enough negative TE to completely switch the background emissivity orderings for detection from the orderings of the previous temperature cases. Recall that it is possible for $T E$ to change sign when $T_{p}<T_{g}$. The detection proportions indicate that $T E$ for Brick may still be positive across the wavenumbers while the $T E$ for Steel-Copper is to small across the wavenumbers to yield any reasonable detections for this temperature case. 


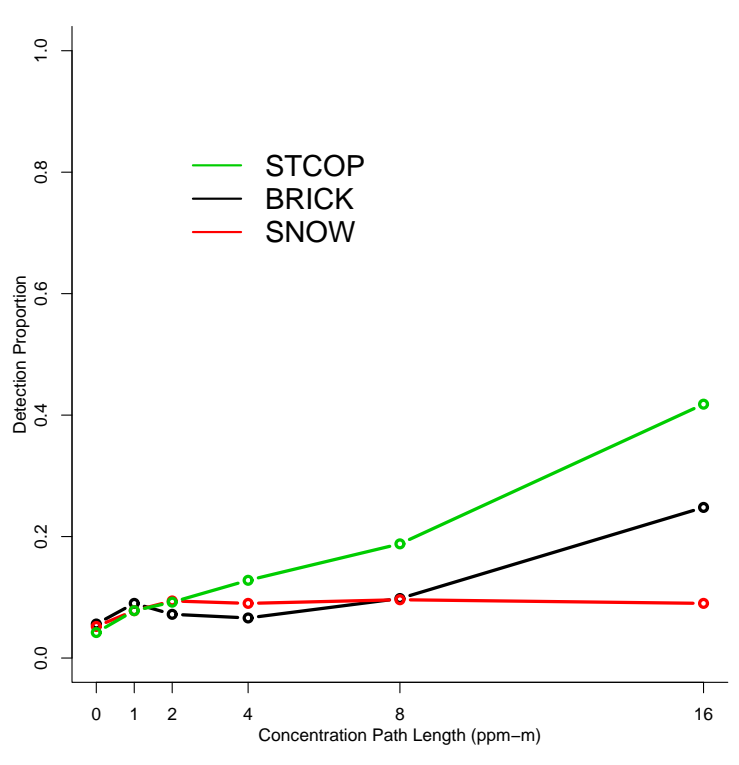

(a)

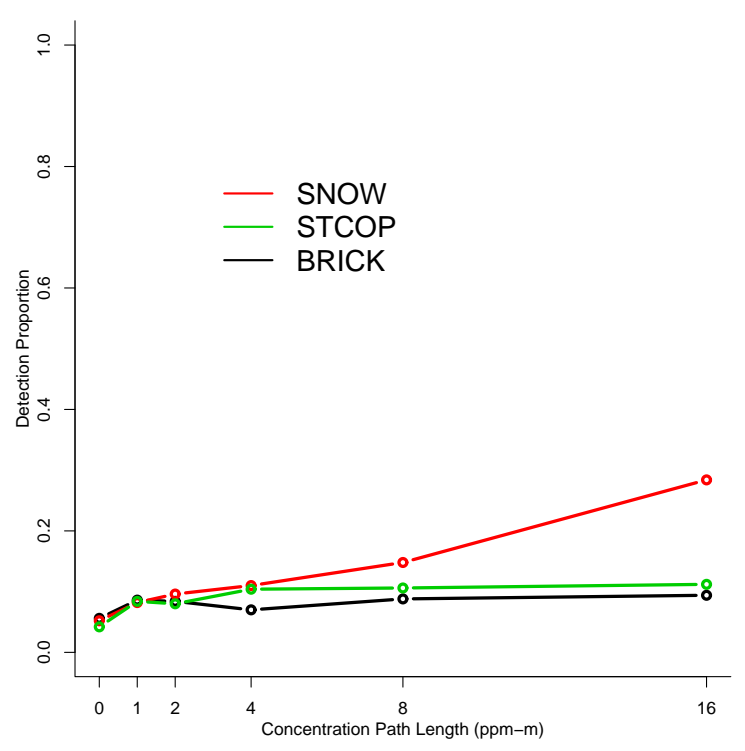

(c)

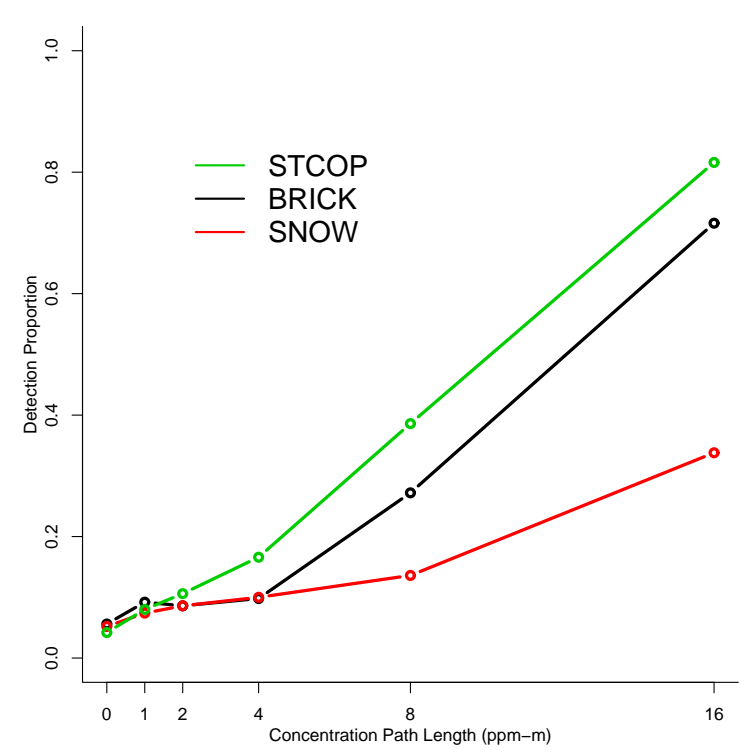

(b)

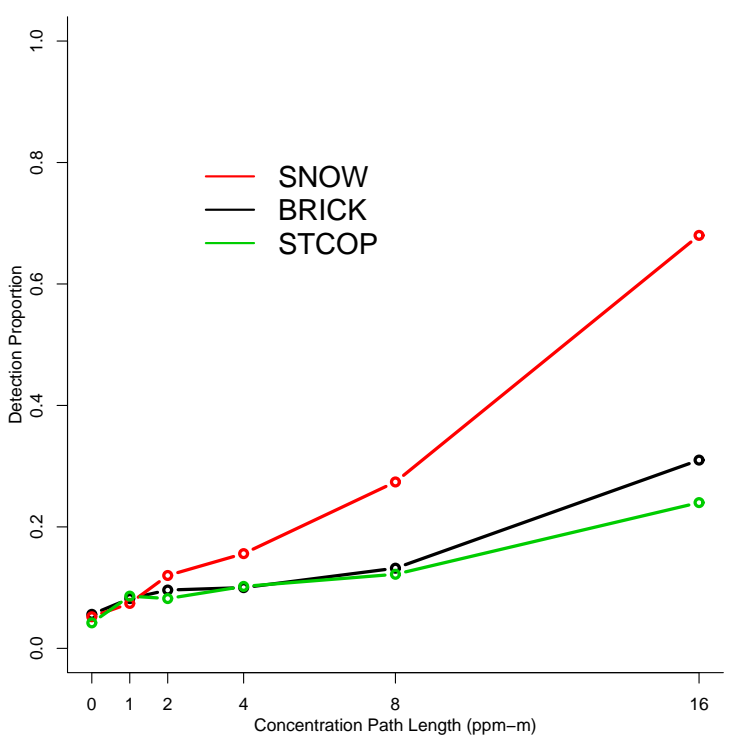

(d)

Figure 6: Detection proportions for $\mathrm{NH}_{3}$ when (a) $T_{p}=T_{g}=300 K$, (b) $T_{p}=305 K>T_{g}=$ $300 K,(\mathrm{c}) T_{p}=295 K<T_{g}=300 K$, and $(\mathrm{d}) T_{p}=290 K<T_{g}=300 K$. 


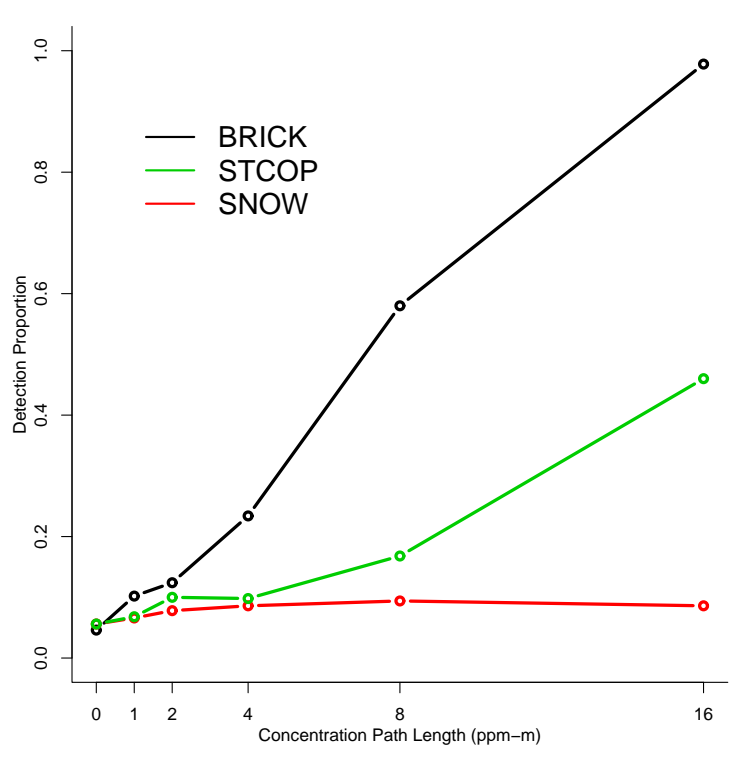

(a)

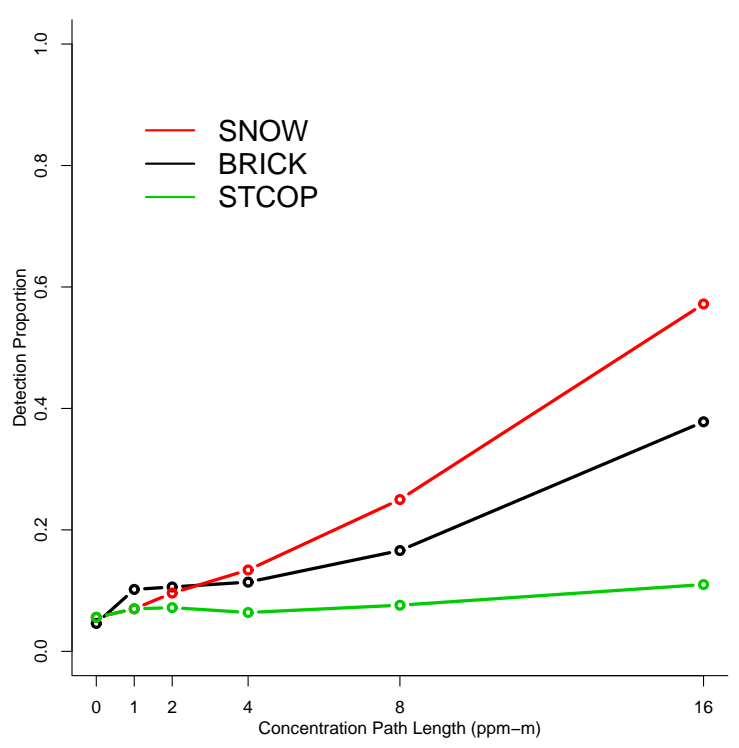

(c)

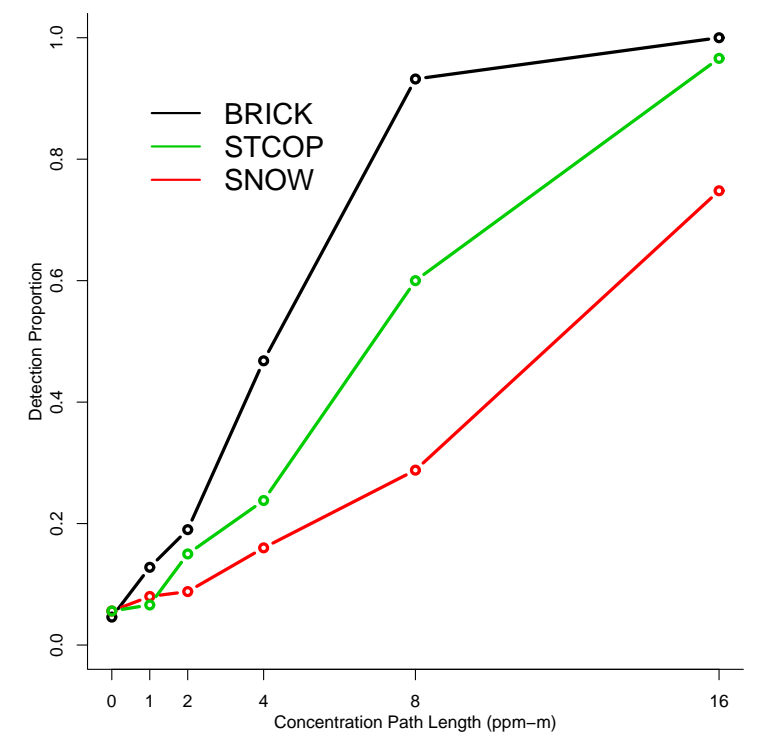

(b)

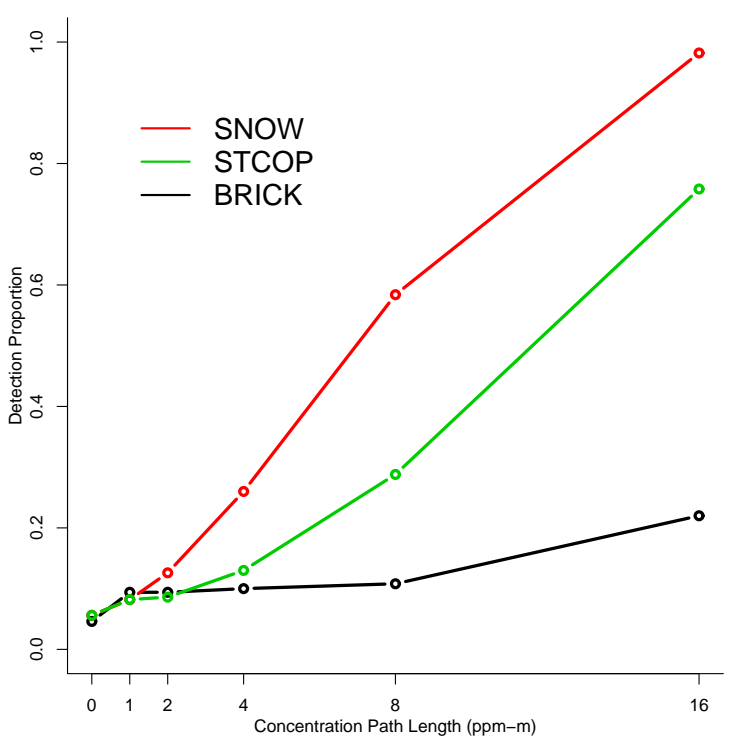

(d)

Figure 7: Detection proportions for TBP when (a) $T_{p}=T_{g}=300 K$, (b) $T_{p}=305 K>T_{g}=$ $300 K,(\mathrm{c}) T_{p}=295 K<T_{g}=300 K$, and $(\mathrm{d}) T_{p}=290 K<T_{g}=300 K$. 
Last, we consider the detection proportion plot for $290=T_{p}<T_{g}=300 \mathrm{~K}$ presented in Figure 7(d). We now observe that the backgrounds can be ordered for best to worst detection of TBP as Snow, Steel-Copper, then Brick. This temperature case was sufficient to give a large enough (negative) $T E$ to observe the switch in background orderings. We also note that, at $1050 \mathrm{~cm}^{-1}$ where TBP exhibits the broad dominant absorbance feature, the background emissivities can be ordered from high to low as Snow, Steel-Copper, then Brick and that this ordering is consistent with the background ordering as decided by the detection proportions. These observations suggest that the large absorbance for TBP is dominating gas detection for this chemical. Further, we note that these observations are consistent with the analysis of $T E$ in Section 2 that when $T_{p}<T_{g}$ larger emissivities yield a larger chemical signal for this temperature case.

\section{Conclusions}

The effects of clutter on gas plume detection/identification is a complicated problem that is approached from multiple perspectives. This paper studied the effects of background emissivity and plume/ground temperatures on the chemical signal. The analysis is most pertinent to treatments of the physical radiance model that linearize the plume transmissivity term and work with linear approaches to gas detection or identification.

Our investigation has shown that, when ignoring reflected downwelling radiance, the physical state of the plume (emission, neutral, or absorption) is not only dependent on the plume/ground temperatures, but is also directly dependent on the background emissivity, $\epsilon_{g}(\nu)$, at a particular wavenumber. We have shown that $T_{p} \geq T_{g}$ implies that the plume is strictly in emission and that values of $\epsilon_{g}(\nu)$ closer to 0 will contribute to a larger observed chemical signal. Further, when $T_{p}<T_{g}$ we have shown that it is possible that the plume is in emission, absorption, or neither emitting nor absorbing (neutral). A neutral plume happens when $\epsilon_{g}(\nu)=B\left(T_{p} ; \nu\right) / B\left(T_{g} ; \nu\right)$. Thus the background has the potential to completely obscure the plume at wavenumber $\nu$. The analysis also shows that emissivities closer to 0 or 1 in this case have the potential to contribute to a larger observed chemical signal.

The analysis was verified by analyzing simulated hyperspectral radiances in the absence of atmospheric, background, and temperature variability. The first two gases considered exhibited a very strong relationship with the background emissivities: the background emissivities 
did not change ordering over the wavenumbers that the gas exhibited absorbance. This made it possible to explore and illustrate the analysis of the physical model in Section 2 that described what types of background emissivities give better chemical detection. The second two gases considered exhibited general variability over the LWIR and results presented with these gases revealed that the dominant gas absorbances may play the greatest role in gas detection. These results also showed that $T_{p}<T_{g}$ is a complex case for gas detection as $T E$ can change signs in this case. These observations warrant further study and we aim to explore this phenomenology in a general setting in future work.

\section{Acknowledgements}

The authors thank Dr. Kevin Anderson for his helpful comments.

\section{References}

[1] T. Burr, B.R. Foy, H. Fry, and B. McVey. Characterizing clutter in the context of detecting weak gaseous plumes in hyperspectral imagery. Sensors, 6:1587-1615, 2006.

[2] T. Burr and N Hengartner. Overview of physical models and statistical approaches for weak gaseous plume detection using passive infrared hyperspectral imagery. Sensors, 6:1721-1750, 2006.

[3] Chein-I Chang, Chao-Cheng Wu, Wei-min Liu, and Yen-Chieh Ouyang. A new growing method for simplex-based endmember extraction algorithm. IEEE Transactions on Geoscience and Remote Sensing., 44(10):2804-2819, 2006.

[4] C. Funk, J. Theiler, D. Roberts, and C. Borel. Clustering to improve matched filter detection of weak gas plumes in hyperspectral thermal imagery. IEEE Trans. Geoscience and Remote Sensing, 39:1410-1420, 2001.

[5] P. Heasler, C. Posse, J. Hylden, and K. Anderson. Nonlinear bayesian algorithms for gas plume detection and estimation from hyper-spectral thermal image data. Sensors, 7:905-920, 2007. 
[6] K.N. Liou. An Introduction to Atmospheric Radiation. Academic Press, 2002, $2^{\text {nd }}$ edition.

[7] D Messinger, C. Salvaggio, and N. Sinisgally. Detection of gaseous effluents from airborned lwir hyperspectral imagery using physics-based signatures. International Journal of High Speed Electronics and Systems., 17(6):801-812, 2007.

[8] J. Neter, M. Kutner, C. Nachtsheim, and W. Wasserman. Applied Linear Statistical Models. McGraw-Hill, 1996, $4^{\text {th }}$ edition.

[9] D. Pogorzala, D. Messinger, and J. Salvaggio, C. Schott. Gas plume species identification by regression analysis. algorithms and technologies for multispectral, hyperspectral, and ultraspectral imagery X,. Proc. SPIE, 5425:583-591, 2004.

[10] J. Schott. Remote Sensing. Oxford Press:New York, 1997.

[11] J. Theiler and B. Foy. Effect of signal contamination in matched-filter detetion of the signal on a cluttered background. IEEE Geoscience and Remote Sensing Letters, 3(1):98-102, 2006.

[12] J. Theiler, B. Foy, and A. Fraser. Characterizing non-gaussian clutter and detecting weak gaseous plumes in hyperspectral imagery. algorithms and technologies for multispectral, hyperspectral, and ultraspectral imagery X,. Proc. SPIE, 5806:182-193, 2005.

[13] J. Wang and Chein-I. Chang. Applications of independent component analysis in endmember extraction and abundance quantification for hyperspectral imagery. IEEE Transactions on Geoscience and Remote Sensing., 44(10):2601-2616, 2006. 